\title{
ON THE IMPORTANCE OF THE INTERCLUMP MEDIUM FOR SUPERIONIZATION: O VI FORMATION IN THE WIND OF $\zeta$ PUPPIS
}

\author{
J. Zsargó, ${ }^{1}$ D. J. Hillier, ${ }^{1}$ J.-C. Bouret, ${ }^{2}$ T. Lanz, ${ }^{3}$ M. A. Leutenegger, ${ }^{4,5}$ And D. H. Cohen ${ }^{6}$ \\ Received 2008 July 15; accepted 2008 August 15; published 2008 August 28
}

\begin{abstract}
We have studied superionization and X-ray line formation in the spectra of $\zeta$ Pup using our new stellar atmosphere code (XCMFGEN) that can be used to simultaneously analyze optical, UV, and X-ray observations. Here, we present results on the formation of the $\mathrm{O}$ vi $\lambda \lambda 1032,1038$ doublet. Our simulations, supported by simple theoretical calculations, show that clumped wind models that assume void in the interclump space cannot reproduce the observed O vi profiles. However, enough O vi can be produced if the voids are filled by a lowdensity gas. The recombination of $\mathrm{O}$ VI is very efficient in the dense material, but in the tenuous interclump region an observable amount of $\mathrm{O}$ VI can be maintained. We also find that different UV resonance lines are sensitive to different density regimes in $\zeta$ Pup: $\mathrm{C}$ IV is almost exclusively formed within the densest regions, while the majority of $\mathrm{O}$ VI resides between clumps. $\mathrm{N} v$ is an intermediate case, with contributions from both the tenuous gas and clumps.
\end{abstract}

Subject headings: stars: early-type — stars: winds, outflows $-\mathrm{X}$-rays: individual ( $\zeta$ Puppis) $-\mathrm{X}$-rays: stars

\section{INTRODUCTION}

One of the surprising discoveries of the Copernicus satellite was the strong P Cygni profiles of superions, such as $\mathrm{O}$ VI and $\mathrm{N} \mathrm{v}$, in the FUV spectra of many O and B stars (Snow \& Morton 1976). The only viable explanation for the presence of $\mathrm{O}$ VI is Auger ionization by X-rays from O IV (Cassinelli \& Olson 1979), which is the dominant form of oxygen in many O-type stars. The X-ray emission, necessary for Auger ionization, was later detected by the first X-ray telescopes (e.g., Harnden et al. 1979; Seward et al. 1979).

The origin of the stellar X-ray emission was another enigma until the "wind-shock" mechanism (Lucy \& White 1980) became the accepted explanation. Massive stars posses strong line-driven winds in which the material is accelerated by numerous $\mathrm{C}, \mathrm{N}, \mathrm{O}$, and Fe transitions (see, e.g., Pauldrach \& Puls 1990; Castor et al. 1975). It was known from the conception of the line-driven wind theory that such flows are unstable and prone to the formation of dense clumps and shocks (see, e.g., Owocki \& Rybicki 1991; Lucy \& White 1980). The large-scale flow energy is converted to heat in the shock fronts, producing high-temperature plasma. Numerical simulations confirm (Feldmeier et al. 1997; Owocki et al. 1988) that at least the soft X-ray emission of early-type stars can be explained by this mechanism.

Evidence for density inhomogeneities (or clumped winds) is provided by variability studies of both W-R stars ( Lépine \& Moffat 1999 and references therein) and O stars (e.g., Eversberg et al. 1998; Lépine \& Moffat 2008). Further, density inhomogeneities allow the electron-scattering wings of emission lines to be reduced to the observed level while maintaining the strength of emission lines (Hillier 1991; Hamann \& Koesterke

\footnotetext{
${ }^{1}$ Department of Physics and Astronomy, University of Pittsburgh, 3941 O'Hara Street, Pittsburgh, PA 15260; jaz8@ itt.edu.

${ }^{2}$ Laboratoire d'Astrophysique de Marseille, CNRS-Université de Provence, 13388 Marseille cedex 13, France.

${ }^{3}$ Department of Astronomy, University of Maryland, College Park, MD 20742.

${ }^{4}$ Goddard Space Flight Center, 8800 Greenbelt Road, Greenbelt, MD 20771.

${ }^{5}$ NASA Postdoctoral Fellow.

${ }^{6}$ Department of Physics and Astronomy, Swarthmore College, 500 College Avenue, Swarthmore, PA 19081.
}

1998; Hillier \& Miller 1999). More recently, Crowther et al. (2002) and Hillier et al. (2003) found that they could not simultaneously fit the $\mathrm{H} \alpha$ and $\mathrm{P} v \lambda \lambda 1120$ profiles in normal $\mathrm{O}$ supergiants without assuming an inhomogeneous density distribution in the wind. Using a more statistical approach, Massa et al. (2003) showed that the phosphorus ionization structure was consistent with expectations only if lower than conventional mass-loss rates were used in their analysis of $\mathrm{P} v \lambda \lambda 1120$. Additional observational evidence for wind clumping comes from Chandra and XMM-Newton high-resolution X-ray spectra of O stars. These spectra revealed that X-ray lines suffer less absorption in the wind than predicted by "smooth" models (e.g., Kramer et al. 2003; Cohen et al. 2006; Waldron \& Cassinelli 2007 and references therein).

Superionization has received only limited attention since the work of MacFarlane et al. (1993, 1994). The effect was introduced into modern stellar atmosphere codes (e.g., WM-basic; Pauldrach et al. 2001), but we are unaware of any work that has revisited the question in light of the high-resolution X-ray observations, improved X-ray emission calculations, and the new results on clumping. With improvements to CMFGEN, we are developing tools and techniques to move toward this goal. As part of this effort we discovered that the interclump medium is crucial to explain the $\mathrm{O}$ vi doublet profile in $\zeta$ Pup. In this Letter we demonstrate the effect and discuss its implications. In $\S 2$ we briefly describe our code, the observations we used, and our models. We present and discuss our results in $\S \S 3$ and 4 .

\section{OBSERVATIONS, TOOLS, AND MODELS}

We co-added Copernicus U1 scans for $\zeta$ Pup to create the observed profile in Figure 1. All but two of these scans were blocked U1 scans that minimize stray light as described in Rogerson et al. (1973a, 1973b). Our data reduction (J. Zsargó \& D. J. Hillier 2008, in preparation) produced an O vi profile that strongly resembles those in MacFarlane et al. (1993) and Morton (1976).

We have used XCMFGEN (J. Zsargó \& D. J. Hillier 2008, in preparation) to solve for the ionization balance and the nonLTE level populations in our stellar models. XCMFGEN is a new version of CMFGEN (Hillier \& Miller 1998) that can 


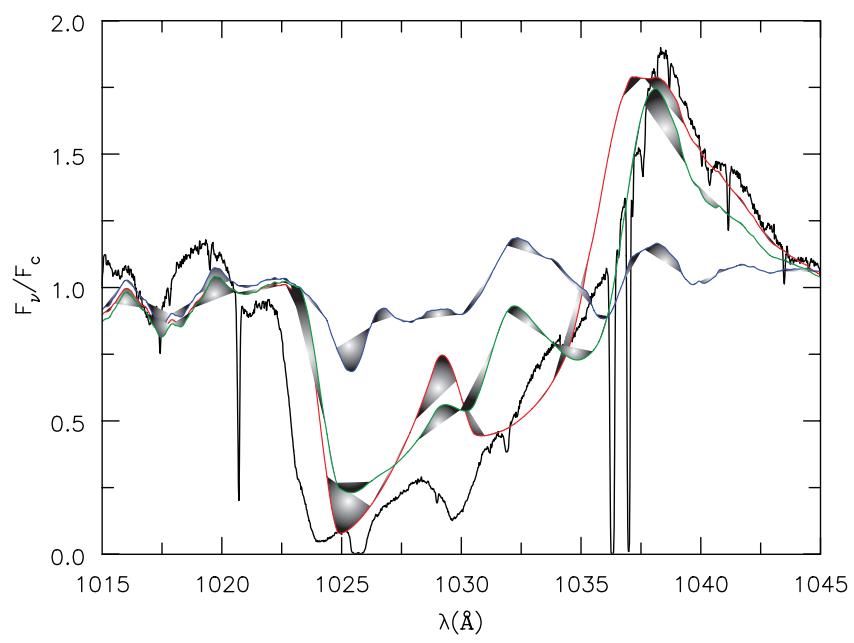

FIG. 1.-Comparison of the Copernicus observation of O vi $\lambda \lambda 1035$ (rough line) with those calculated by XCMFGEN for a smooth wind ( $r e d$ ), for a clumped wind model (blue), and for the interclump medium (green). See Table 1 and $\S$ 3 for details.

perform X-ray emission calculations in addition to the original CMFGEN tasks. We use APEC (Smith et al. 2001) and its accompanying database of X-ray cross sections (APED) to calculate non-LTE level populations and emissivities in the X-rayemitting plasma.

Bouret et al. (2005; J. Bouret et al. 2008, in preparation) used CMFGEN to derive stellar parameters for seven O supergiants. We used their values for $\zeta$ Pup to construct the models listed in Table 1. Our improved models specifically addressed formation of $\mathrm{O}$ VI and X-ray lines (J. Zsargó \& D. J. Hillier 2008 , in preparation). The X-ray-emitting plasma was distributed in the wind, and its emissivity was characterized by three plasma temperatures. The parametrization included a volume filling factor $\left(f_{\mathrm{X}}\right)$ and an initial turn-on radius $\left(R_{0}\right)$, as in Owocki $\&$ Cohen (2001). The volume filling factor was constant beyond $R_{0}$ and was adjusted until the prescribed $L_{\mathrm{X}} / L_{\mathrm{BOL}}$ was met (see Table 1). Note that $L_{\mathrm{X}}$ is the $\mathrm{X}$-ray luminosity attenuated by the wind and not the intrinsic luminosity.

To treat clumping we follow the "volume filling factor" approach where the material is compressed into a fraction of the available volume with void in between (see, e.g., Hillier \& Miller 1999; Hamann \& Koesterke 1998). The ratio of the volume filled with material (clumps) to the total is

$$
f_{\mathrm{cl}}(r)=f_{\infty}+\left(1-f_{\infty}\right) \exp \left[\frac{-v(r)}{v_{\mathrm{cl}}}\right],
$$

where $f_{\infty}$ and $v_{c l}$ are free parameters. Equation (1) is an ad hoc formula, motivated by hydrodynamical simulations, and provides a smooth wind at low $v(r)$ velocities $\left(r \sim R_{*}\right)$.

In our models, $f_{\mathrm{x}}$ and $f_{\mathrm{cl}}$ are two independent parameters despite the fact that both are referred to as "filling factors." The two should be related since both of them are the results of the wind instability. However, our understanding of the linedriven winds is too poor to formulate this relationship.

\section{RESULTS AND DISCUSSION}

Figure 1 shows our model calculations together with the Copernicus spectrum. Auger ionization produces strong O VI features in the case of the smooth wind model, albeit a bit weaker than observed. A better fit can be achieved by increas-
TABLE 1

Model Parameters (Representative for $\zeta$ Pup)

\begin{tabular}{|c|c|}
\hline Photosphere/Wind & Value \\
\hline 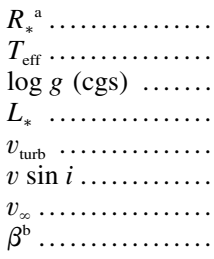 & $\begin{array}{c}19 R_{\odot} \\
39,000 \mathrm{~K} \\
3.6 \\
7.0 \times 10^{5} L_{\odot} \\
12 \mathrm{~km} \mathrm{~s}^{-1} \\
240 \mathrm{~km} \mathrm{~s}^{-1} \\
2300 \mathrm{~km} \mathrm{~s}^{-1} \\
0.9\end{array}$ \\
\hline & Clumped Wind \\
\hline $\begin{array}{l}\dot{M} \ldots \ldots \ldots \ldots \ldots \\
f_{\infty} \ldots \ldots \ldots \ldots \ldots \ldots\end{array}$ & $\begin{array}{cc}7.6 \times 10^{-6} M_{\odot} \mathrm{yr}^{-1} & 1.7 \times 10^{-6} M_{\odot} \mathrm{yr}^{-1} \\
\ldots & 0.05 \\
\ldots & 150 \mathrm{~km} \mathrm{~s}^{-1} \\
\end{array}$ \\
\hline \multicolumn{2}{|l|}{ Plasma } \\
\hline $\begin{array}{l}R_{0}{ }^{\mathrm{c}} \ldots \ldots \ldots \ldots \ldots \\
L_{\mathrm{X}} / L_{\mathrm{BOL}} \ldots \ldots \ldots \ldots \ldots \\
T_{\mathrm{pl}} 1^{\mathrm{a}} \ldots \ldots \ldots \ldots \ldots \\
T_{\mathrm{pl}} 2 \ldots \ldots \ldots \ldots \ldots \\
T_{\mathrm{pl}} 3^{\mathrm{a}} \ldots \ldots \ldots \ldots \ldots .\end{array}$ & $\begin{array}{l}1.5 R_{*} \\
1.5 \times 10^{-7} \\
4.6 \times 10^{6} \mathrm{~K} \\
2.4 \times 10^{6} \mathrm{~K} \\
1.7 \times 10^{6} \mathrm{~K}\end{array}$ \\
\hline
\end{tabular}

NoTE. - Stellar parameters are from J. Bouret et al. (2008, in preparation) unless noted otherwise. Plasma parameters are from J. Zsargó \& D. J. Hillier (2008, in preparation) unless noted otherwise. For simplicity, the same emission measure $\mathrm{EM}_{\mathrm{X}}=$ $\int n_{e} n_{\mathrm{H}} d V$ was assumed for each $T_{\mathrm{pl}}$.

${ }^{a}$ Value is from Hillier et al. (1993).

${ }^{\mathrm{b}}$ Power of the CAK velocity law (Castor et al. 1975).

${ }^{c}$ Value is from Kramer et al. (2003) and Leutenegger et al. (2006).

ing the oxygen abundance and by assuming larger turbulent velocities near $v_{\infty}$. A detailed analysis of $\zeta$ Pup and the simultaneous fit to the X-ray and UV spectra will be presented in J. Zsargó \& D. J. Hillier (2008, in preparation). The most important feature of Figure 1 is the absence of an $\mathrm{O}$ vi profile in the clumped wind model. This cannot be rectified by modifying, for example, the oxygen abundance. Adjustments at the order-of-magnitude level would be necessary to produce a visible $\mathrm{O}$ VI profile; this indicates that something fundamental is wrong with the simplified "clumped" model.

There is a simple theoretical explanation for the weak O VI P Cygni profiles in clumped models. Since the $O$ VI $\lambda \lambda 1032$, 1038 doublet forms only in the wind by redistribution of the stellar radiation, the optical depth controls the shape and strength of the profiles (see, e.g., Lamers et al. 1987). In a spherical and accelerating flow, it is appropriate to write the optical depth as

$$
\tau_{\mathrm{OVI}} \sim \frac{\pi e^{2}}{m c} \frac{f n_{\mathrm{OVI}} \lambda_{0}}{(v / r)+[(d v / d r)-(v / r)] \mu^{2}},
$$

where the atomic parameters have the usual meanings and $\arccos (\mu)$ is the angle between a line of sight and the radial direction. The average $\mathrm{O}$ VI density can be written as

$$
n_{\mathrm{OVI}}=A_{\mathrm{O}} n q_{\mathrm{OVI}} \sim A_{\mathrm{O}} \frac{\dot{M}}{4 \pi r^{2} v(r) m_{p}} q_{\mathrm{ovI}}
$$

by using the oxygen abundance $A_{\mathrm{O}}$, the $\mathrm{O}$ VI fraction $q_{\mathrm{ov}}$, and the average particle mass $m_{p}$. Note that $n_{\mathrm{OVI}}$ is the mean ion density over a Sobolev length, so

$$
n_{\mathrm{OVI}}=n_{\mathrm{OVI}, \mathrm{cl}} f_{\mathrm{cl}}=A_{\mathrm{O}} n_{\mathrm{cl}} f_{\mathrm{cl}} q_{\mathrm{OVI}}
$$


must be used if the wind material occupies only a fraction $f_{\mathrm{cl}}$ of the available volume with $n_{\mathrm{cl}}$ number density.

The key unknown in the expression for $\tau_{\mathrm{O}}{ }_{\mathrm{VI}}$ is the $\mathrm{O}$ VI fraction. We can estimate $q_{\mathrm{O} \text { VI }}$ by a simplified rate equation:

$$
\frac{d n_{\mathrm{OVI}}}{d t}=n_{\mathrm{OIV}} \alpha\left(J_{\mathrm{X}}\right)-n_{\mathrm{OVI}} n_{e} \gamma=0
$$

where $\alpha\left(J_{\mathrm{X}}\right)$ is the $\mathrm{X}$-ray flux-dependent rate for Auger ionization and $\gamma$ is the effective recombination rate coefficient. Solving for the ion fraction,

$$
\frac{n_{\mathrm{OVI}}}{n_{\mathrm{OIV}}}=\frac{q_{\mathrm{OVI}}}{q_{\mathrm{OIV}}}=\frac{\alpha\left(J_{\mathrm{X}}\right)}{n_{e} \gamma}
$$

Under the approximation that $q_{\mathrm{OIV}} \sim 1$ and $n_{e} \sim n$, we find that $q_{\mathrm{ovI}}=\alpha\left(J_{\mathrm{X}}\right) / n \gamma$, or

$$
n_{\mathrm{OVI}}=A_{\mathrm{O}} n q_{\mathrm{OVI}}=A_{\mathrm{O}} \frac{\alpha\left(J_{\mathrm{X}}\right)}{\gamma}
$$

The ion density of $\mathrm{O}$ VI, therefore, is independent of the wind density and thus the mass-loss rate. Substituting this in the expression for the optical depth, we find for a smooth wind

$$
\tau_{\mathrm{OVI}, \mathrm{sm}} \sim \frac{\pi e^{2}}{m c} \frac{A_{\mathrm{O}} \alpha\left(J_{\mathrm{X}}\right)}{\gamma} \frac{f \lambda_{0}}{(v / r)+[(d v / d r)-(v / r)] \mu^{2}} .
$$

The Sobolev optical depth in a smooth wind is thus also independent of the mass-loss rate.

Let us generalize this to a clumped wind. Equations (6) and (7) still give the O VI fraction and density, but these are the values for the dense clumps and not means over a Sobolev length. Thus, we need to use equations (2), (4), and (6), together with the assumptions of $q_{\mathrm{O} \text { IV }} \sim 1$ and $n_{e} \sim n$, to derive

$$
\tau_{\mathrm{OVI}, \mathrm{cl}} \sim f_{\mathrm{cl}} \tau_{\mathrm{OVI}, \mathrm{sm}}
$$

in a clumped wind.

It is clear now why clumped models fail to produce observable $\mathrm{O}$ vi lines. The volume filling factor for $\zeta$ Pup $\left(f_{\mathrm{cl}} \sim 0.05\right.$ at $\left.r \gg R_{*}\right)$ decreases $\tau_{\mathrm{O} \mathrm{VI}, \mathrm{cl}}$ well below unity. The results of our XCMFGEN simulations fully support the above calculation. Figure 2 shows the radial optical depth of the important UV resonance lines in our models (Table 1). The curves show what is predicted by equation (9) for small $f_{\mathrm{cl}}$; the $\mathrm{O} \mathrm{VI}$ optical depth is nearly 2 orders of magnitude lower than in the smooth wind model. The dense clumps, therefore, contribute little or nothing to the $\mathrm{O}$ vi profiles.

There are two other factors that further decrease the $\mathrm{O}$ VI optical depth and hence adversely influence the model O VI profile. First, J. Bouret et al. (2008, in preparation) found subsolar oxygen abundances for all stars in their sample (a factor of 5 difference for $\zeta$ Pup); this affects only comparisons with earlier calculations. Second, the X-ray flux available for Auger ionization tends to decrease when $f_{\mathrm{cl}}$ is lowered; the wind absorption is smaller, hence less $\mathrm{X}$-ray emission is needed to meet the observed $L_{\mathrm{X}} / L_{\mathrm{BOL}}$.

What are the implications of our failure to produce observable $\mathrm{O}$ vi lines with clumped winds? Is the wind smoother than suggested by J. Bouret et al. (2008, in preparation)? Their filling factor for $\zeta$ Pup is not extremely low; it is very similar to those

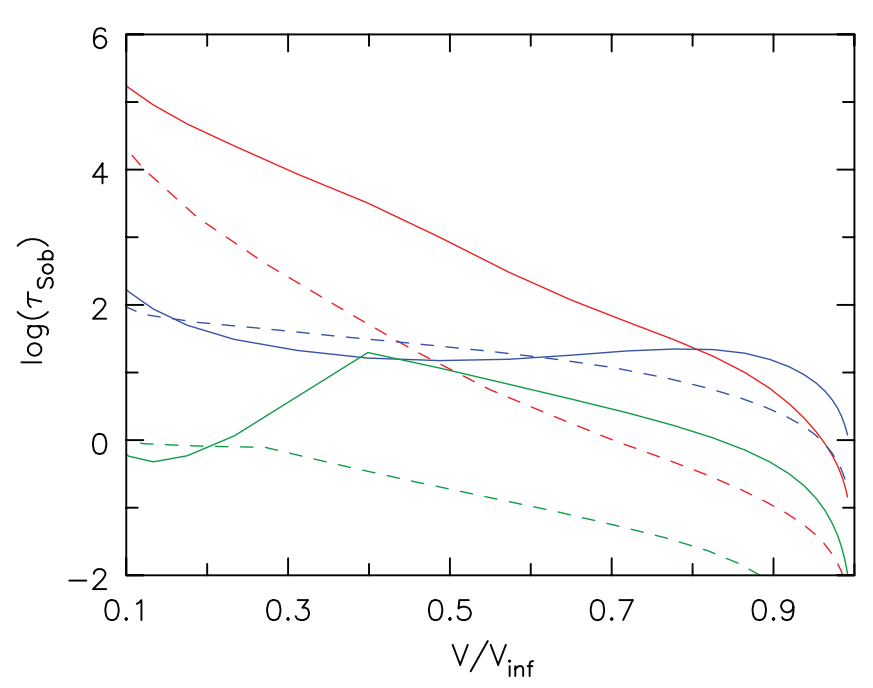

FIG. 2.-Radial optical depth as a function of normalized wind velocity for the stronger component of the $\mathrm{N} \vee \lambda \lambda 1240$ (red), C IV $\lambda \lambda 1550$ (blue), and $\mathrm{O}$ vi $\lambda \lambda 1035$ (green) doublets. The solid and dashed lines are for smooth and clumped wind models (Table 1), respectively.

found for other O supergiants (Hillier et al. 2003; Massa et al. 2003). Further, increasing $f_{\mathrm{cl}}$ to 0.1 or 0.2 would not solve the problem. It is more likely that the use of the traditional "filling factor" approach in XCMFGEN causes the failure to produce observable $\mathrm{O}$ VI lines. Equations (8) and (9) suggest that the part of the wind with the largest filling factor will be the most important at producing $\mathrm{O}$ vI optical depth. Thus, we need to consider the influence of the tenuous interclump medium (ICM) on the $\mathrm{O}$ vi profile.

This is a nontrivial exercise; at least three components (dense clumps, ICM, and the hot plasma) need to be treated simultaneously for a fully self-consistent solution. Fortunately, the radiation field at any radius is almost independent of the ICM. Therefore, we can use $J_{v}(R)$ from our clumped model and solve the statistical equilibrium equations in the ICM only. We created such a model from the clumped model of Table 1 . We scaled down the densities by a factor of $f_{\mathrm{cl}}^{2}$ to simulate the ICM at $r \gg R_{*}$ and imported the radiation field from the original clumped model. We also assumed that the new model is smooth, reflecting the high volume filling fraction of the ICM. Effectively, we took the ICM component out of the real (clump + ICM + hot plasma) wind and built a stand-alone model for it. Since we imported the radiation field from the clumped model, the coupling between the ICM and the rest of the wind has been taken into account at least in the first order.

The choice of density in the ICM is somewhat arbitrary, but in the absence of reliable hydrodynamical predictions it is still reasonable. There is a density contrast of 400 between the dense clumps and the ICM at $r \gg R_{*}$. This means that the ICM contributes little to the overall mass-loss rates. If we combined the models for the ICM and clumps, the total $\dot{M}$ would be nearly identical (within 5\%) to that of the clumped model.

The result of this experiment is displayed in Figures 1 and 3. It is obvious from both figures that the ICM has substantial O VI optical depth and contributes a strong P Cygni profile. Our results also offer a glimpse of the behavior of other lines. The $\mathrm{C}$ IV optical depth in the ICM is very low, and thus most of the $C$ IV $\lambda \lambda 1550$ lines are produced by the clumps. The behavior of $\mathrm{N} v \lambda \lambda 1240$ is very interesting. It appears from Figure 3 that both the ICM and the dense medium contribute. 


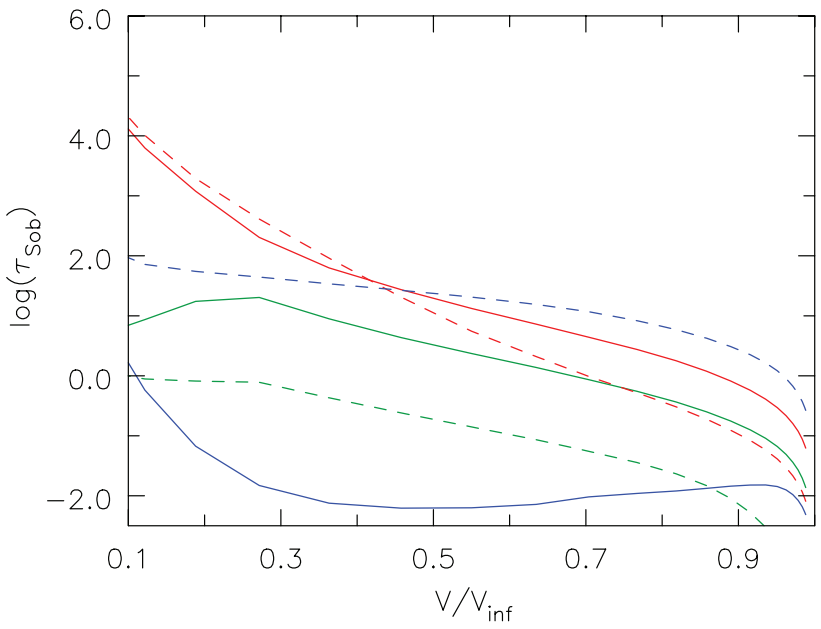

FIg. 3.-Same as Fig. 2, but the solid curves are now for the interclump medium. See $\S 3$ for details.

Our results warrant an investigation of the physical conditions in the ICM and also its role in the formation of UV resonance lines in different environments (e.g., low- and highdensity winds). For example, what is the density contrast between the ICM and clumps? At very low densities the average charge state of oxygen (and other species) may be drastically different from the one in the dense regions. Also, how closely does the ICM follow the velocity of the clumps? Do O VI and C IV follow a different velocity structure? Further, how are the ICM and hot plasma related?

\section{CONCLUSION}

In this Letter we present our first results on superionization in clumped winds and showcase the potential of the interclump medium to produce observable features. Clumped wind models that use the classical "volume filling factor" approach (clumps with voids in between) cannot reproduce the observed $\mathrm{O}$ VI profile in $\zeta$ Pup. The recombination of $\mathrm{O} \mathrm{VI}$ is too efficient and the necessary fractional abundance cannot be sustained in the clumps. However, a tenuous interclump medium can contribute enough $\mathrm{O}$ VI to produce an observable $\mathrm{O}$ VI profile. Only a small amount of mass is necessary in the interclump medium, so its overall effect on the derived mass-loss rates is negligible.

Our result highlights the need for improved treatment of clumping in the winds of massive stars. It is impossible to achieve a simultaneous fit to all UV P Cygni profiles with a single-component wind model for $\zeta$ Pup. Our simulations suggest that in $\zeta$ Pup, different UV resonance lines probe different density regimes. C IV is formed almost exclusively in the dense material, while $\mathrm{O}$ vi likely originates from the interclump medium. $\mathrm{N} \mathrm{v}$ is an intermediate case with similar contributions from both components. In cooler $\mathrm{O}$ stars, when $\mathrm{N}^{2+}$ become the dominant ionization stage, we might expect that $\mathrm{N} v$ shows the same behavior as $\mathrm{O}$ VI in the hotter stars. Obviously other possible effects of the interclump medium, in both $\mathrm{O}$ and W-R stars, should be investigated.

This research was supported by STScI grant HST-AR10693.02 and by SAO grant TM6-7003X. We are also grateful to Randall Smith for providing us the source code of APEC and to the Chandra X-Ray Center for the use of ATOMDB. Maurice A. Leutenegger acknowledges support from a fellowship administered by Oak Ridge Associated Universities under the NASA Postdoctoral Program. J.-C. Bouret acknowledges financial support from the French National Research Agency (ANR) through program ANR-06-BLAN-0105. David Cohen acknowledges support from grant AR7-8002X from the Chandra X-Ray Center at SAO.

\section{REFERENCES}

Bouret, J.-C., Lanz, T., \& Hillier, D. J. 2005, A\&A, 438, 301

Cassinelli, J. P., \& Olson, G. L. 1979, ApJ, 229, 304

Castor, J., Abbott, D. C., \& Klein, R. I. 1975, ApJ, 195, 157

Cohen, D. H., et al. 2006, MNRAS, 368, 1905

Crowther, P. A., et al. 2002, ApJ, 579, 774

Eversberg, T., Lépine, S., \& Moffat, A. F. J. 1998, ApJ, 494, 799

Feldmeier, A., Puls, J., \& Pauldrach, A. W. A. 1997, A\&A, 322, 878

Hamann, W.-R., \& Koesterke, L. 1998, A\&A, 335, 1003

Harnden, F. R., Jr., et al. 1979, ApJ, 234, L51

Hillier, D. J. 1991, A\&A, 247, 455

Hillier, D. J., \& Miller, D. L. 1998, ApJ, 496, 407 1999, ApJ, 519, 354

Hillier, D. J., et al. 1993, A\&A, 276, 117

2003, ApJ, 588, 1039

Kramer, R. H., Cohen, D. H., \& Owocki, S. P. 2003, ApJ, 592, 532

Lamers, H. J. G. L. M., Cerruti-Sola, M., \& Perinotto, M. 1987, ApJ, 314, 726

Lépine, S., \& Moffat, A. F. J. 1999, ApJ, 514, 909 . 2008, AJ, 136, 548
Leutenegger, M. A., Paerels, F. B. S., Kahn, S. M., \& Cohen, D. H. 2006, ApJ, 650, 1096

Lucy, L. B., \& White, R. L. 1980, ApJ, 241, 300

Macfarlane, J. J., Cohen, D. H., \& Wang, P. 1994, ApJ, 437, 351

MacFarlane, J. J., et al. 1993, ApJ, 419, 813

Massa, D., Fullerton, A. W., Sonneborn, G., \& Hutchings, J. B. 2003, ApJ, 586, 996

Morton, D. C. 1976, ApJ, 203, 386

Owocki, S. P., Castor, J. I., \& Rybicki, G. B. 1988, ApJ, 335, 914

Owocki, S. P., \& Cohen, D. H. 2001, ApJ, 559, 1108

Owocki, S. P., \& Rybicki, G. B. 1991, ApJ, 368, 261

Pauldrach, A. W. A., Hoffmann, T. L., \& Lennon, M. 2001, A\&A, 375, 161

Pauldrach, A. W. A., \& Puls, J. 1990, A\&A, 237, 409

Rogerson, J. B., et al. 1973a, ApJ, 181, L97 . 1973b, ApJ, 181, L110

Seward, F. D., et al. 1979, ApJ, 234, L55

Smith, R. K., Brickhouse, N. S., Liedahl, D. A., \& Raymond, J. C. 2001, ApJ, 556, L91

Snow, T. P., \& Morton, D. C. 1976, ApJS, 32, 429

Waldron, W. L., \& Cassinelli, J. P. 2007, ApJ, 668, 456 